\title{
Sondas de Discriminação na Avaliação da Aprendizagem de Relações Emergentes Nome-Objeto por Exclusão
}

\author{
Leylanne Martins Ribeiro de Souza*,1 \\ Orcid.org/0000-0002-6175-3183 \\ Maria Stella Coutinho de Alcantara Gil ${ }^{1}$ \\ Orcid.org/0000-0003-4375-3232 \\ Lucas Tadeu Garcia ${ }^{1}$ \\ Orcid.org/0000-0003-2897-9356
}

${ }^{1}$ Universidade Federal de São Carlos, São Carlos, SP, Brasil

\begin{abstract}
Resumo
O responder por exclusão é um padrão de resposta robusto, entretanto, a ocorrência regular da resposta que atesta a relação condicional nome-objeto não corresponde à aprendizagem consistente da relação. Este estudo visou avaliar a aprendizagem de relações nome-objeto, após a emergência da relação condicional no responder por exclusão, empregando sondas de discriminação as quais foram expostas 19 crianças, entre 14 e 25 meses. Discriminações condicionais nome-objeto com estímulos familiares foram ensinadas para compor a linha de base. Sondas controle, de exclusão e de discriminação verificaram respectivamente o controle pela novidade dos estímulos, a emergência da relação nomeobjeto e a aprendizagem da relação emergente. Se não ocorresse a aprendizagem da relação emergente, inseria-se o ensino por exclusão. Seis participantes responderam por exclusão. Sondas de discriminação atestaram a aprendizagem de duas relações nome-objeto para três participantes. Um participante respondeu consistentemente com a aprendizagem de duas relações, após o ensino da relação nomeobjeto emergente. As sondas de discriminação constituíram-se em medida efetiva da aprendizagem de relações emergentes nome-objeto por crianças pequenas.
\end{abstract}

Palavras-chave: Responder por exclusão, aprendizagem por exclusão, sondas de discriminação, crianças.

* Endereço para correspondência: Universidade Federal de São Carlos, Departamento de Psicologia, Centro de Educação e Ciências Humanas, Jardim Guanabara, São Carlos, SP, Brasil 13565-905. Caixa-postal: 676. Fone: (16) 3351-8493. E-mail: leylannemrs@yahoo.com.br,dpsi.stellagil@gmail.com e lucas.uai@gmail.com Apoio: Coordenação de Aperfeiçoamento de Pessoal de Nível Superior(CAPES), processo 88887.136407/201700; Conselho Nacional de Desenvolvimento Científico e Tecnológico (CNPq), processo 465686/2014-1; e Fundação de Amparo à Pesquisa do Estado de São Paulo (FAPESP), processo 2014/50909-8, em apoio ao Instituto Nacional de Ciência e Tecnologia sobre Comportamento, Cognição e Ensino (INCT-ECCE). 


\title{
Discrimination Probes for Evaluating Learning of Emergent Name-Object Relations by Exclusion
}

\begin{abstract}
Responding by exclusion is a robust response pattern; however, regular occurrence of responses that attest the name-object conditional relation does not correspond to consistent learning of the relation. This study aimed to evaluate learning of name-object relations, after emergence of the conditional relation in responding by exclusion, through discrimination probes presented to 19 children between 14 and 25 months old. Familiar name-object conditional stimuli were taught as baseline. Control, exclusion, and discrimination probes found control by stimulus novelty, emergence of name-object relation, and learning of emergent relation, respectively. If the learning of the emergent relation did not occur, the teaching by exclusion was inserted. Six participants responded by exclusion. Discrimination probes attested learning of two name-object relations by three participants. One participant's responses were consistent with learning two relations after teaching of emergent name-object relations. Discrimination probes were found to be an effective measure for learning of emergent name-object relations by small children.
\end{abstract}

Keywords: Responding by exclusion, learning by exclusion, discrimination probes, children.

\section{Pruebas de Discriminación en la Evaluación del Aprendizaje de Relaciones Emergentes Nombre-Objeto por Exclusión}

\section{Resumen}

La respuesta por exclusión es un patrón de respuesta robusta. Sin embargo, la ocurrencia regular de la respuesta que da cuenta de la relación condicional nombre-objeto no se corresponde al aprendizaje consistente de dicha relación. Este estudio tuvo como objetivo evaluar el aprendizaje de relaciones nombre-objeto, después de la emergencia de la relación condicional al responder por exclusión, utilizando ensayos de prueba de discriminación a las cuales fueron expuestos 19 niños, de entre 14 y 25 meses. Discriminaciones condicionales nombre-objeto con estímulos familiares se enseñaron para constituir la línea base. Las pruebas de control, exclusión y discriminación verificaron, respectivamente, el control por novedad de los estímulos, la emergencia de la relación nombre-objeto y el aprendizaje de la relación emergente. Si no ocurría el aprendizaje de la relación emergente, se inserta la enseñanza por exclusión. Seis participantes respondieron por exclusión. Las pruebas de discriminación mostraron el aprendizaje de las relaciones nombre-objeto para tres participantes. Un participante respondió consistentemente con el aprendizaje de dos relaciones, después de la enseñanza de la relación nombre-objeto emergente. Las pruebas de discriminación se constituyeron como una medida efectiva del aprendizaje de relaciones emergentes nombre-objeto para niños pequeños.

Palabras clave: Responder por exclusión, aprendizaje por exclusión, pruebas de discriminación, niños.

O responder por exclusão se refere à escolha de um estímulo com base na rejeição de outro(s) estímulo(s). No contexto das investigações sobre aquisição de vocabulário, o termo tem sido utilizado para se referir à escolha de um objeto ou referente indefinido diante de um modelo (e.g., palavra) também indefinido, quando aquele é apresentado simultaneamente a um ou mais estímulos definidos. Esse padrão de escolha tem sido documentado consistentemente na literatura experimental, tanto em humanos como em animais (e.g., Dixon, 1977; Wilkinson, Dube, \& McIl- 
vane, 1998). A expressão "estímulo definido" se refere aos estímulos (modelos ou comparações) cuja relação com outro estímulo foi estabelecida dentro ou fora do contexto experimental; "estímulo indefinido" se refere aos estímulos não previamente relacionados a qualquer outro estímulo. Por sua vez, um estímulo novo se refere aos estímulos com os quais os participantes nunca interagiram, ou que estão sendo apresentados pela primeira vez durante o experimento.

Um importante problema de pesquisa na área, entretanto, decorre da inconsistência da manutenção do controle de estímulos emergente na tentativa de exclusão em contextos subsequentes. Especialmente no que tange a aprendizagem de palavras ou de relações auditivo-visuais (e.g., nome-objeto ou nome-figura), estudos recentes com crianças pequenas (entre 18 e 36 meses) têm indicado que uma única exposição a tentativas de exclusão raramente é suficiente para que os participantes aprendam a nova relação (Domeniconi, Costa, de Souza, \& de Rose, 2007; Horst \& Samuelson, 2008; Ribeiro de Souza, Gil, \& Garcia, 2018; Ribeiro de Souza, Minto de Sousa, \& Gil, 2016; Schmidt, Franco, Lotério, \& Gomes, 2016).

Para investigar o que tem sido denominado "aprendizagem por exclusão", em tarefas de emparelhamento com o modelo, os pesquisadores têm utilizado um procedimento no qual, após o ensino de discriminações condicionais de linha de base, os participantes são expostos a sondas de exclusão, nas quais um modelo indefinido é apresentado e o participante deve escolher um estímulo indefinido o qual é apresentado simultaneamente a um ou mais estímulos de comparação definidos. Em caso de escolha do estímulo indefinido, a avaliação da aprendizagem da relação entre modelo e comparação indefinidos é conduzida em seguida, geralmente por meio de diferentes tipos de arranjo de tentativas de sonda (c.f., Costa, Domeniconi, \& de Souza, 2014).

No estudo seminal de Dixon (1977), por exemplo, após os participantes (adolescentes com deficiência intelectual) terem escolhido consistentemente as letras upsilon e theta diante de seus respectivos nomes, por exclusão, eles foram apresentados a tentativas nas quais apenas as duas letras e seus nomes se alternavam como comparações e modelos, respectivamente. A autora chamou estas sondas de tentativas de discriminação. Naquele estudo, a despeito da consistência da escolha por exclusão, apenas dois de oito participantes apresentaram um desempenho consistente com aprendizagem para o primeiro conjunto de estímulos (letras gregas). Para os demais conjuntos, os participantes apresentaram um desempenho consistente: seis de oito participantes atingiram critério para o segundo conjunto, de letras japonesas; e no terceiro conjunto, de letras gregas, seis participantes pontuaram acima do critério estabelecido no pré-teste e os outros dois participantes atingiram critério.

Com a finalidade de investigar os fatores que influenciavam tanto a escolha por exclusão quanto o desempenho nas sondas de aprendizagem, alguns autores desenvolveram diferentes arranjos de tentativas que permitem avaliar as topografias de controle de estímulo que controlam o responder dos participantes nas tarefas (Wilkinson \& McIlvane, 1997). Para isso, um dos recursos mais utilizados foi a inserção entre os estímulos comparação, do chamado comparação vazio, ou máscara, que permite, por exemplo, avaliar se a escolha por exclusão ocorre mesmo na ausência de um estímulo indefinido novo na matriz de estímulos (Costa, Wilkinson, McIlvane, \& de Souza, 2001). A máscara pode ser designada como escolha incorreta, ou correta (quando substitui o estímulo relacionado ao modelo apresentado) em uma tentativa e permite a rejeição de todos os outros estímulos apresentados. Neste procedimento, a máscara permite identificar o controle experimental dos efeitos de escolha pela novidade ou preferência por um estímulo.

Costa et al. (2014) descreveram os quatro principais tipos de sondas de aprendizagem utilizadas nos estudos sobre exclusão. Estas sondas têm a função de avaliar se o controle da escolha do estímulo indefinido diante do mesmo modelo se mantém em situações posteriores, nas quais os parâmetros que possibilitaram a exclusão não estão mais presentes. As avaliações podem re- 
querer que o indivíduo novamente selecione o estímulo diante do mesmo modelo apresentado anteriormente, em um contexto com estímulos novos ou distratores, ou ainda que o indivíduo rejeite o estímulo escolhido na sonda por exclusão, estabelecendo uma nova relação por exclusão.

$\mathrm{Na}$ sonda de aprendizagem 1 (modelo: estímulo indefinido novo; comparações: estímulo definido, indefinido presente na exclusão e máscara), a escolha correta depende da rejeição do estímulo escolhido na sonda de exclusão diante do novo estímulo modelo e do estímulo definido, resultando na escolha da máscara. Na sonda de aprendizagem 2 (modelo: estímulo indefinido novo; comparações: estímulo indefinido presente na exclusão, indefinido novo e máscara), uma escolha correta ocorrerá com base na seleção de um novo estímulo de comparação indefinido diante de um novo modelo, assim como da rejeição da máscara e do estímulo escolhido na sonda de exclusão. Na sonda de aprendizagem 3 (modelo: estímulo indefinido presente na exclusão; comparações: estímulo definido, indefinido novo e máscara), o participante deve rejeitar um estímulo definido e um novo estímulo indefinido diante do modelo da sonda de exclusão, escolhendo a máscara. E na sonda de aprendizagem 4 (modelo: estímulo indefinido presente na exclusão; comparações: estímulo indefinido presente na exclusão, indefinido novo e máscara), a escolha correta deve ser realizada com base na seleção do estímulo indefinido escolhido na sonda de exclusão diante do modelo correspondente, e a rejeição de um novo estímulo indefinido e da máscara.

Estas sondas, muitas vezes utilizadas em diferentes combinações para avaliar a aprendizagem, produziram resultados importantes para esclarecer as fontes de controle de estímulo que produzem responder e aprendizagem consistentes por exclusão, em crianças a partir dos 24 meses de idade (Costa et al., 2001; Wilkinson \& McIlvane, 1997). Com base em um critério de acertos em ao menos três daquelas sondas, as evidências de aprendizagem em crianças abaixo dos 36 meses de idade, depois de apenas uma escolha por exclusão, têm sido escassas (Anto- niazzi, Domeniconi, \& Schmidt, 2014; Barbosa, Gomes, Costa, \& Schmidt, 2015; Domeniconi et al., 2007; Ribeiro, Gallano, Souza, \& de Souza, 2016; Ribeiro de Souza et al., 2016; Schmidt et al., 2016).

Estes resultados desafiadores têm justificado as hipóteses sobre quais fatores levam à aprendizagem rápida de novas relações condicionais ou, mais especificamente, de novas palavras. Alguns fatores estariam relacionados às características dos participantes, tais como a idade ou a extensão do vocabulário; outros se referem a aspectos do procedimento, por exemplo, a quantidade de tentativas de exclusão, o tipo de atividade, entre outros (Bion, Borovsky, \& Fernald, 2013; Horst \& Samuelson, 2008; Langsdorff, Schmidt, \& Domeniconi, 2015; Schmidt et al., 2016). Entretanto, alguns resultados sugerem que o desempenho de crianças mais jovens poderia estar relacionado a certas características dos arranjos dos estímulos de comparação nas sondas tradicionais de aprendizagem (Ribeiro de Souza et al., 2018; Schmidt et al., 2016), como por exemplo, controle por rejeição.

O principal fato que sustenta esse argumento é de que algumas sondas (por exemplo, sondas com controle por rejeição) têm produzido maior proporção de erros do que outras. As tentativas que os participantes mais erram são aquelas que requerem a rejeição dos estímulos de comparação indefinidos mais novos - sondas tipo 3 e 4 (c.f., Costa et al., 2014; Ribeiro de Souza et al., 2016; Schmidt et al., 2016). Embora o arranjo que possibilite a rejeição de estímulos indefinidos mais novos seja importante para garantir que a relação nova aprendida seja avaliada com base em diferentes topografias de controle de estímulo, fortalecendo os parâmetros para inferência de aprendizagem, é possível que um viés de escolha com base na novidade possa afetar os resultados observados. Algumas evidências sobre os padrões de escolha de crianças em situações de aprendizagem de relações entre nome-objeto apontam nessa direção (c.f., Horst, Samuelson, Kucker, \& McMurray, 2011).

Se o arranjo de estímulos nas tentativas de avaliação de aprendizagem pode aumentar a 
probabilidade de erro dos participantes, então, as evidências negativas sobre aprendizagem por exclusão em crianças pequenas podem estar sendo produzidas pelos métodos de avaliação utilizados, especialmente em função do controle pela novidade decorrente da inserção de estímulos indefinidos novos nas sondas. Consequentemente, é importante avaliar se outras metodologias produzem resultados distintos. Uma alternativa seria empregar as sondas de discriminação utilizadas em muitos dos estudos iniciais sobre o responder por exclusão (e.g., Carr, 2003; de Rose, de Souza, \& Hanna, 1996; de Rose, de Souza, Rossito, \& de Rose, 1989; Dixon, 1977; McIlvane \& Stoddard, 1981). Nas sondas de discriminação, a aprendizagem por exclusão é avaliada em tentativas nas quais apenas os estímulos apresentados nas sondas de exclusão se alternam como modelos e comparações, ou seja, não são utilizados novos estímulos indefinidos. Outra alternativa seria recorrer a medidas adicionais que poderiam servir como indicativo de aprendizagem, tais como as respostas de nomeação emergentes da escolha por exclusão (e.g., Costa, Grisante, Domeniconi, de Rose, \& de Souza, 2013). Ainda que, especialmente para crianças pequenas, a emergência de respostas de nomeação não necessariamente ocorra depois da aprendizagem por exclusão (Costa et al., 2013; Greer \& Du, 2015; Ribeiro et al., 2016), sua ocorrência presumivelmente sugere que a relação nome-objeto tenha sido aprendida.

Dessa forma, este estudo visou avaliar a aprendizagem por exclusão de relações entre nomes e objetos por meio de sondas de discriminação (Dixon, 1977), após a emergência da relação condicional no responder por exclusão, em 19 participantes com idades entre 14 e 25 meses. Além disso, objetivou avaliar se os resultados positivos nestas sondas corresponderiam aos resultados positivos em dois outros tipos de metodologias de avaliação de aprendizagem por exclusão: três sondas de aprendizagem (tipo 1, 3 e 4) comumente utilizadas na literatura da área (e.g., Costa et al., 2014) e sondas de nomeação (Costa et al., 2013).

\section{Método}

\section{Participantes}

Participaram 19 crianças com idade entre 14 a 25 meses no início do experimento (10 meninos e nove meninas), recrutadas em uma creche.

\section{Instrumentos}

O repertório inicial e final das crianças foi avaliado por meio de dois instrumentos. $\mathrm{O}$ Teste de Triagem Denver II (Frankenburg, Dodds, Archer, \& Bresnick, 1990; versão traduzida e adaptada para o português por Pedromônico, Bragatto, \& Strobilus, 1999) e o Inventário Portage Operacionalizado, IPO ("Portage Checklist", Bluma, Shearer, Frohman, \& Hilliard, 1976, versão traduzida e adaptada para o português por Williams \& Aiello, 2001). Os dois instrumentos empregados propiciaram o levantamento do repertório de crianças a partir de 0 meses e permitiram a reaplicação mês a mês.

\section{Materiais e Equipamentos}

Os estímulos visuais eram seis brinquedos do cotidiano dos participantes e oito brinquedos construídos com feltro. Os estímulos auditivos eram os nomes dos brinquedos do cotidiano (/ auau/, /bola/, /carro/, /cubo/, /palhacinho/, /pocotó/) e pseudopalavras para os brinquedos de feltro (/fapi/; /beva/; /daga/; /búgu/; /mido/). As pseudopalavras /fapi/ e /beva/ foram retiradas dos estudos de Ribeiro et al. (2016) por estarem em consonância com a aquisição fonológica típica da faixa etária dos participantes (Lamprecht et al., 2004). Três dos brinquedos de feltro eram expostos, mas nunca nomeados. Brinquedos diversos foram empregados nas brincadeiras ao final das sessões.

Os brinquedos eram expostos em um "caderno de ensino" com adaptações (Almeida, 2014). Consistia em um conjunto de 13 folhas de papel cartão preto, encadernadas por uma espiral. Folhas contendo três bolsos plásticos transparentes para apresentação dos objetos eram intercaladas com páginas pretas, expostas no intervalo entre tentativas. As páginas mediam $65 \mathrm{~cm}$ de largura x $35 \mathrm{~cm}$ de altura, e os bolsos plásticos transpa- 
rentes mediam $18 \mathrm{~cm}$ largura x $24 \mathrm{~cm}$ de altura, distando $3 \mathrm{~cm}$ entre si, a $10 \mathrm{~cm}$ da margem superior e a $1 \mathrm{~cm}$ da margem inferior. Para registro das sessões foi utilizada uma filmadora digital Sony ${ }^{\circledR}$ Cyber-Shot DSC W610 14.1MP, que focalizava a experimentadora, o participante e o caderno de ensino.

\section{Situação Experimental}

As sessões experimentais ocorreram em uma sala da creche com área de $10 \mathrm{~m}^{2}$, localizada próxima aos berçários.

\section{Procedimento}

Coleta de dados. As sessões tinham a duração média de quatro minutos e ocorreram em média quatro vezes por semana, em um período entre cinco e oito meses. A experimentadora e participante permaneciam sentados no chão, frente a frente, com o caderno de ensino ou os brinquedos entre eles. Encerradas as tarefas experimentais, havia dois minutos de brincadeira livre (Gil, Oliveira, de Sousa, \& Faleiros, 2006) com brinquedos diferentes dos estímulos experimentais. Previamente à coleta de dados houve um período de quatro semanas para a familiarização entre a pesquisadora e os participantes.

O procedimento constituiu-se de três fases: Fase 1 - ensino das discriminações condicionais de linha de base; Fase 2 - ensino da resposta à máscara, ensino da linha de base com a máscara e sondas controle; Fase 3 - sondas de exclusão e aprendizagem. A avaliação da aprendizagem foi realizada por meio de três procedimentos: sondas de discriminação; sondas de aprendizagem; e sondas de nomeação. $\mathrm{O}$ critério de aprendizagem da relação emergente era de $100 \%$ de acertos nas sondas de discriminação. As sondas posteriores de aprendizagem e nomeação foram utilizadas como medidas adicionais de retenção, devido à sua consistência na literatura da área (c.f., Costa et al., 2014). Caso o critério não fosse atingido, era realizada uma quarta fase que consistia de um bloco de ensino por exclusão (com reforçamento diferencial) seguido das sondas de discriminação, nomeação e aprendizagem.

Um procedimento de correção foi empregado para os casos de ausência de resposta de sele- ção. O modelo era ditado a cada três segundos, durante 30 segundos, até que a criança tocasse um dos bolsos. Se nenhuma resposta ocorresse, uma nova tentativa era iniciada. Escolhas condizentes com o modelo eram seguidas de elogios e acesso ao objeto por cerca de $60 \mathrm{~s}$. Escolhas não condizentes com o modelo eram seguidas de silêncio da experimentadora e restrição do acesso ao objeto. Se o participante emitisse dois erros, na seleção dos estímulos de comparação, a sessão era encerrada.

As sessões de ensino de linha de base eram compostas de seis tentativas, com o acerto nas tentativas produzindo reforço em esquema de reforçamento contínuo (CRF) ou razão variável 2 (VR2), e o critério de aprendizagem era de seis acertos em seis tentativas; ou cinco acertos em seis tentativas, em duas sessões consecutivas. Os blocos de sondas eram programados com oito tentativas, com exceção da sonda controle. Todas as tentativas de sonda (controle, exclusão, discriminação, aprendizagem e nomeação) foram realizadas em extinção e o critério de aprendizagem era o acerto em todas as tentativas de sondas da sessão.

Fase 1 - Estabelecimento das discriminações condicionais com estímulos familiares (linha de base - LB). As tentativas iniciavam com a apresentação de um modelo por meio da seguinte instrução: "(Nome da criança), pegue $o$ (nome do objeto)". Em seguida, os estímulos de comparação eram apresentados no caderno de ensino (exposição de um, dois e três estímulos de comparação). Escolhas (apontar ou tocar) do estímulo designado como correto eram seguidas de elogios e acesso ao objeto por cerca de 60 segundos, em CRF. Escolhas incorretas eram seguidas de um período de silêncio da experimentadora com restrição do acesso do participante ao objeto, e o início de uma nova tentativa. A sessão era encerrada se o participante emitisse dois erros distribuídos ao longo da sessão.

Na primeira etapa do ensino, cada uma das relações nome-objeto era ensinada separadamente em sessões nas quais apenas um estímulo modelo e um comparação ( $\mathrm{S}+$ ) era apresentado. Em seguida, o mesmo procedimento era realizado, mas em cada tentativa eram apresentados 
Tabela 1

Delineamento Experimental

\begin{tabular}{|c|c|c|c|c|c|c|}
\hline Fases & Tarefa & $\begin{array}{c}\mathrm{N}^{\mathrm{o}} \\
\text { tentativas }\end{array}$ & Modelo ${ }^{\mathrm{a}, \mathrm{b}}$ & $\mathrm{N}^{\mathrm{o}} \mathrm{Cos}$ & $\begin{array}{l}\text { Esquema de } \\
\text { reforçamento }\end{array}$ & $\begin{array}{c}\text { Critério } \\
(\%)\end{array}$ \\
\hline & Linha de base & 18 & $\mathrm{D} 1, \mathrm{D} 2, \mathrm{D} 3$ & 1 & $\mathrm{CRF}$ & 100 \\
\hline \multirow{2}{*}{$\begin{array}{c}1 \\
\text { Sem máscara }\end{array}$} & Linha de base & 6 & $\mathrm{D} 1, \mathrm{D} 2, \mathrm{D} 3$ & 2 & $\mathrm{CRF}$ & 100 ou $83(2 x)$ \\
\hline & Linha de base & 6 & D1,D2,D3 & 3 & $\mathrm{CRF}$ & 100 ou $83(2 x)$ \\
\hline \multirow{10}{*}{$\begin{array}{c}2 \\
\text { Com máscara }\end{array}$} & Ensino de resposta máscara & 18 & D1,D2,D3 & 1 & $\mathrm{CRF}$ & 100 \\
\hline & Linha de base & 6 & $\mathrm{D} 1, \mathrm{D} 2, \mathrm{D} 3$ & 2 & $\mathrm{CRF}$ & 100 ou $83(2 x)$ \\
\hline & Linha de base & 6 & D1,D2,D3 & 3 & $\mathrm{CRF}$ & 100 ou $83(2 x)$ \\
\hline & Linha de base & 6 & D1,D2,D3 & 3 & VR2 & 100 ou $83(2 x)$ \\
\hline & Sonda & & & & & \\
\hline & Linha de base & 4 & $\mathrm{D} 1, \mathrm{D} 2, \mathrm{D} 3$ & 3 & VR2 & 100 \\
\hline & Controle & 2 & D1, D3 & 3 & Ext & 100 \\
\hline & Sonda & & & & & \\
\hline & Linha de base & 4 & $\mathrm{D} 1, \mathrm{D} 2, \mathrm{D} 3$ & 3 & VR2 & 100 \\
\hline & Controle & 2 & I5 & 3 & Ext & 100 \\
\hline \multirow{8}{*}{$\begin{array}{c}3 \\
\text { Com máscara }\end{array}$} & Sonda & & & & & \\
\hline & Linha de base & 2 & $\mathrm{D} 1, \mathrm{D} 2, \mathrm{D} 3$ & 3 & $\mathrm{CRF}$ & 100 \\
\hline & Exclusão & 2 & $\mathrm{I} 1 ; \mathrm{I} 2$ & 3 & Ext & 100 \\
\hline & Discriminação & 2 & I1; I2 & 3 & Ext & 100 \\
\hline & Nomeação & 2 & $\mathrm{I} 1 ; \mathrm{I} 2$ & & Ext & \\
\hline & Sonda & & & & & \\
\hline & Linha de base & 2 & D1,D2,D3 & 3 & $\mathrm{CRF}$ & 100 \\
\hline & Aprendizagem & 6 & $\mathrm{I} 1 ; \mathrm{I} 2 ; \mathrm{I} 3 ; \mathrm{I} 4$ & 3 & Ext & \\
\hline \multirow{9}{*}{$\begin{array}{c}4 \\
\text { Com máscara }\end{array}$} & Ensino & & & & & \\
\hline & Linha de base & 2 & D1, D3 & 3 & VR2 & 100 \\
\hline & Exclusão & 6 & I1; I2 & 3 & $\mathrm{CRF}$ & 100 \\
\hline & Nomeação & 2 & I1; I2 & & Ext & \\
\hline & Sonda & & & & & \\
\hline & Linha de base & 1 & $\mathrm{D} 1, \mathrm{D} 2, \mathrm{D} 3$ & 3 & $\mathrm{CRF}$ & 100 \\
\hline & Discriminação & 2 & I1; I2 & 3 & Ext & 100 \\
\hline & Aprendizagem & 6 & $\mathrm{I} 1 ; \mathrm{I} 2 ; \mathrm{I} 3 ; \mathrm{I} 4$ & 3 & Ext & \\
\hline & Nomeação & 2 & I1; I2 & & Ext & \\
\hline
\end{tabular}

Nota. $\mathrm{N}^{\mathrm{o}}$ tentativas=número de tentativas; $\mathrm{N}^{\mathrm{o}} \mathrm{Cos}=$ número de estímulos comparação; $\mathrm{CRF}=$ reforçamento contínuo; VR2= razão variável 2; Ext=extinção.

${ }^{a}$ Estímulos definidos $-\mathrm{D} 1=$ auau; D2= carro; D3= bola.

${ }^{\mathrm{b}}$ Estímulos indefinidos $-\mathrm{I} 1=$ fapi; $\mathrm{I} 2=$ beva; $\mathrm{I} 3=$ daga; $\mathrm{I} 4=$ búgu; $\mathrm{I} 5=$ mido. 
dois estímulos comparações (o $\mathrm{S}+$ e um dos S-'s). Por fim, era realizada uma sessão na qual os três estímulos modelo e de comparação se alternavam ao longo das tentativas (duas tentativas para cada modelo). A quantidade de estímulos de comparação era aumentada gradativamente. Se o participante não atingisse o critério em três sessões, o conjunto de estímulos era substituído (/cubo/, /palhacinho/, /pocotó/).

Fase 2 - Ensino de resposta à máscara. $\mathrm{O}$ objetivo desta etapa era ensinar os participantes a escolher bolso plástico opaco, como comparação vazio (Sertori, 2013). O procedimento de fading in da máscara foi realizado pelo acréscimo gradual de folhas de papel semitransparente entre o bolso plástico e o objeto, tornando-o mais opaco ao longo de seis tentativas. $\mathrm{O}$ ensino foi realizado em três sessões de seis tentativas para cada estímulo (um estímulo modelo e um estímulo de comparação), em CRF. O critério de aprendizagem era de seis acertos em seis tentativas.

Ensino das relações de linha de base (LB) com máscara. Nesta etapa as relações ensinadas na Fase 1 foram apresentadas em sessões de ensino nas quais a máscara era apresentada como estímulo de comparação. As tentativas iniciavam-se com a apresentação de um modelo auditivo (instrução) e, no intervalo de 2 segundos, os estímulos de comparação eram apresentados no caderno de ensino (dois e três estímulos de comparação); em todas as sessões, o comparação vazio (máscara) substituía o $\mathrm{S}+$ em metade das tentativas. Foi ensinada a discriminação entre duas relações, em CRF. Após atingido o critério, eram apresentadas sessões em CRF com as três relações de linha de base. Depois que o participante atingia o critério, era realizada uma sessão em razão variável 2 como preparação para as sondas.

Sondas controle. Ao final do ensino da linha de base era realizada uma sessão de seis tentativas composta de quatro tentativas de LB e duas tentativas de sondas controle. A primeira sonda controle avaliava a ocorrência de controle pela novidade. Era apresentado um modelo definido da LB (MD1 ou MD3) e os estímulos de comparação eram: um objeto definido, um objeto indefinido novo (CI7 ou CI8) e a máscara.
Respostas ao $\mathrm{CI} 7$ ou CI8 sugeriam um controle pela novidade. A segunda sonda controle verificava se a máscara era efetivamente uma alternativa de escolha para os participantes. O modelo era uma palavra ditada indefinida (MI5, /mido/) e os estímulos de comparação eram dois objetos definidos e a máscara.

Fase 3 - Sondas de exclusão, discriminação, nomeação e aprendizagem. As sessões de sondas eram compostas de oito tentativas. Todas as tentativas de linha de base eram reforçadas, enquanto as sondas eram realizadas em extinção. Na primeira sessão de sonda, eram apresentadas duas tentativas de LB, duas sondas de exclusão, duas sondas de discriminação entre as relações novas e duas sondas de nomeação. $\mathrm{O}$ acerto nas sondas de exclusão era condição para a exposição às demais sondas. A segunda sessão era composta por: duas tentativas de LB, seis sondas de aprendizagem tradicionais e duas sondas de nomeação. $O$ critério utilizado para atestar a ocorrência de aprendizagem por exclusão foi a escolha correta nas duas sondas de discriminação da primeira sessão, entretanto, para fins de comparação, as sondas de aprendizagem e nomeação foram utilizadas como medidas adicionais.

Sondas de exclusão. Nas tentativas de exclusão o modelo era uma palavra indefinida (MI1 ou MI2, /fapi/ ou /beva/), e os comparações eram um objeto definido, um objeto indefinido (CI1 ou CI2) e a máscara. Respostas aos objetos indefinidos eram consideradas responder por exclusão. Escolhas da máscara levavam ao encerramento da sessão e a realização de uma nova sessão em que a máscara era substituída por outro objeto definido, para aumentar a probabilidade de escolha do estímulo indefinido e permitir a avaliação da aprendizagem.

Sondas de discriminação. As sondas de discriminação apresentavam a estrutura originalmente utilizada por Dixon (1977), nas quais apenas os estímulos novos (apresentados nas sondas de exclusão) eram apresentados como modelos ou comparação. Nestas tentativas, o modelo era uma das duas palavras indefinidas apresentadas na sonda de exclusão (MI1 ou MI2, /fapi/ ou / beva/) e os estímulos de comparação eram os 
dois objetos indefinidos (CI1 e CI2) e a máscara. Escolhas do comparação correspondente ao modelo indicariam aprendizagem.

Sondas de nomeação. Ao final da sessão de sondas de discriminação, eram realizadas duas tentativas, uma para cada estímulo indefinido, /fapi/ e /beva/. Nestas tentativas, o objeto indefinido era apresentado pela experimentadora acompanhado da instrução: "(Nome da criança), qual é o nome dele?". O objetivo era verificar se as respostas de tato (Skinner, 1957) diante dos objetos emergiriam. Respostas correspondentes ponto a ponto com o nome do estímulo indefinido (MI) eram consideradas corretas.

Sondas de aprendizagem. A estrutura e nome das sondas foram baseadas na classificação fornecida por Costa et al. (2014). A primeira sonda (Tipo 4) apresentava como modelo o estímulo da sonda de exclusão (MI1 ou MI2), e os estímulos de comparação eram o objeto escolhido na sonda de exclusão (CI1 ou CI2), um objeto novo (CI3 ou CI4) e a máscara. Respostas aos estímulos CI1 ou CI2 foram consideradas consistentes com a aprendizagem da relação de emparelhamento palavra/figura, com base no controle de seleção (relação modelo-S + ).

$\mathrm{Na}$ segunda sonda realizada (Tipo 3), o estímulo modelo da sonda de exclusão era apresentado (MI1 ou MI2), e os estímulos de comparação eram um estímulo definido (CD), um segundo estímulo indefinido (CI7 ou CI8) e a máscara. Respostas à máscara foram consideradas indicativo de aprendizagem. Em seguida, outra sonda de aprendizagem (Tipo 1) apresentava um segundo estímulo indefinido como modelo (MI3 ou MI4) e os estímulos de comparação eram um estímulo definido (CD), o estímulo escolhido na sonda de exclusão (CI1 ou CI2) e a máscara. A seleção da máscara sugeria a rejeição do estímulo indefinido escolhido na sonda de exclusão (supostamente já conhecido na ocasião da exclusão) e formar uma nova relação (nome-objeto novos). Nessas sondas, a aprendizagem da relação de emparelhamento palavra/figura seria demonstrada com base no controle de rejeição (relação modelo-S-).

Após essa sessão, se o critério de aprendizagem nas sondas de discriminação tivesse sido atingido, o experimento era encerrado para aquele participante. Caso contrário, iniciava-se os procedimentos de ensino por exclusão (Tabela 1).

Fase 4 - Ensino por exclusão. O objetivo desta fase era verificar se a apresentação de reforço contingente às escolhas por exclusão promoveria a aprendizagem das novas relações auditivo-visuais em crianças que não a demonstraram previamente (e.g., Carr, 2003). A sessão de ensino por exclusão era composta por 10 tentativas: duas tentativas de LB, seis tentativas de exclusão e duas sondas de nomeação. As tentativas de LB eram conduzidas em VR2, as tentativas de ensino por exclusão eram em CRF e as tentativas de nomeação eram em extinção.

Em seguida, era realizada uma sessão de sondas, composta por nove tentativas: uma tentativa de LB (em CRF), duas sondas de discriminação e seis sondas de aprendizagem (todas em extinção). $\mathrm{O}$ critério de aprendizagem das relações emergentes era de $100 \%$ de acertos nas sondas de discriminação. Se o critério fosse atingido, eram realizadas duas sondas de nomeação, em extinção. Se o critério não fosse atingido as sessões de ensino por exclusão eram novamente realizadas, até o número máximo de 3 repetições.

Análise de dados. Os dados foram analisados pelo cálculo da porcentagem de escolhas corretas dos participantes durante o ensino da linha de base, ou com base nos parâmetros descritos de escolhas indicativas de exclusão ou aprendizagem, bem como, de controle pela novidade. As respostas de nomeação foram analisadas com base na correspondência ponto-a-ponto entre a resposta verbal do participante diante do objeto e as palavras faladas utilizadas como modelo.

Acordo entre observadores. Trinta por cento dos registros das sessões ( 73 sessões, 337 tentativas) foram analisados por dois observadores independentes para estabelecer um índice de concordância entre observadores. O cálculo foi realizado dividindo o número de tentativas em que houve acordo pelo número de acordos somado ao número de desacordos, multiplicado por 100 (c.f., Kazdin, 1982), referente às respostas de escolha dos participantes. A porcentagem de concordância entre os observadores foi de $100 \%$. 
Avaliação da integridade do comportamento do aplicador na execução do procedimento. Em 73 sessões, dois observadores examinaram a compatibilidade dos comportamentos do aplicador em relação ao plano experimental no que diz respeito a: instruções para os participantes, estímulos de comparação apresentados em cada tentativa e consequências liberadas diante do acerto ou erro emitido pelo participante. O cálculo realizado foi o mesmo para as respostas de escolha dos participantes (c.f., Kazdin, 1982). A porcentagem de concordância entre os observadores para os comportamentos do aplicador foi de $97 \%$.

\section{Procedimentos Éticos}

O projeto foi aprovado pelo Comitê de Ética em Pesquisa em Seres Humanos da Universidade Federal de São Carlos (Parecer 1.088.954 de 09/06/2015), seguindo as recomendações da Resolução 466/2012. A diretora da creche autorizou a realização da pesquisa. A participação das crianças foi autorizada pelos pais ou responsáveis, que assinaram o Termo de Consentimento Livre e Esclarecido (TCLE). Os pais foram informados sobre os objetivos da pesquisa e as tarefas que as crianças realizariam com a pesquisadora.

\section{Resultados}

No Teste de Triagem Denver II adaptado para o português (Pedromônico et al., 1999), 11 participantes apresentaram indicativos de risco para o desenvolvimento: três (P3, P6 e P11) no fator linguagem, três (P1, P5 e P11) no fator pessoal-social, e oito (P1, P9, P11, P12, P13, P14, $\mathrm{P} 15$ e P16) no fator motor-grosseiro na avaliação inicial. No Teste de Triagem Denver II final, oito apresentaram indicativos de risco para o desenvolvimento: dois participantes (P3 e P11) no fator linguagem e oito (P1, P3, P5, P7, P8, P9, P10 e P11) no fator motor-grosseiro, ver Tabela 2. Os demais apresentaram desenvolvimento típico.

$\mathrm{Na}$ avaliação inicial do Inventário Portage Operacionalizado (Williams \& Aiello, 2001), para as áreas de linguagem e cognição houve desempenho abaixo de $80 \%$ em relação ao desempenho estimado pelo IPO para todos os participantes. Na avaliação final do IPO, o desempenho manteve-se abaixo de $80 \%$ para 16 participantes na área de linguagem (P1, P2, P3, P4, P5, P6, P7, P8, P9, P10, P11, P12, P13, P14, P15 e P16), e para 14 participantes na área da cognição (P1, P2, P3, P4, P5, P6, P7, P8, P9, P10, P11, P12, $\mathrm{P} 13$ e P16). Entretanto, houve aumento na pontuação de cada um dos participantes da primeira para a última avaliação conforme consta da Tabela 2 .

Seis participantes (P6, P14, P15, P17, P18 e P19) responderam por exclusão. Sondas de discriminação atestaram a aprendizagem de duas relações nome-objeto para três participantes (P6, P14 e P19). Um participante (P17) respondeu consistentemente com a aprendizagem de duas relações no ensino da relação nome-objeto, após uma sonda de exclusão e três tentativas similares à tentativa de exclusão. Os participantes não demonstraram aprendizagem nas medidas adicionais - sondas de aprendizagem $(1,3$ e 4$)$ e de nomeação.

\section{Fase 1 - Estabelecimento das Discriminações Condicionais com Estímulos Familiares (linha de base - LB)}

Sete dos 19 participantes (P5, P6, P14, P15, P17, P18 e P19) atingiram os critérios de aprendizagem das três relações condicionais auditivovisuais de linha de base. O desempenho desses participantes nas tentativas de LB permaneceu acurado no decorrer das outras fases do procedimento. Todos os participantes atingiram o critério na primeira etapa do treino de linha de base, em que apenas um estímulo modelo era apresentado. Durante o ensino com dois comparações, 11 participantes atingiram o critério. Na sessão com três estímulos de comparação, quatro desses 11 participantes ( $\mathrm{P} 4, \mathrm{P} 8, \mathrm{P} 9$ e $\mathrm{P} 13)$ não atingiram critério. 


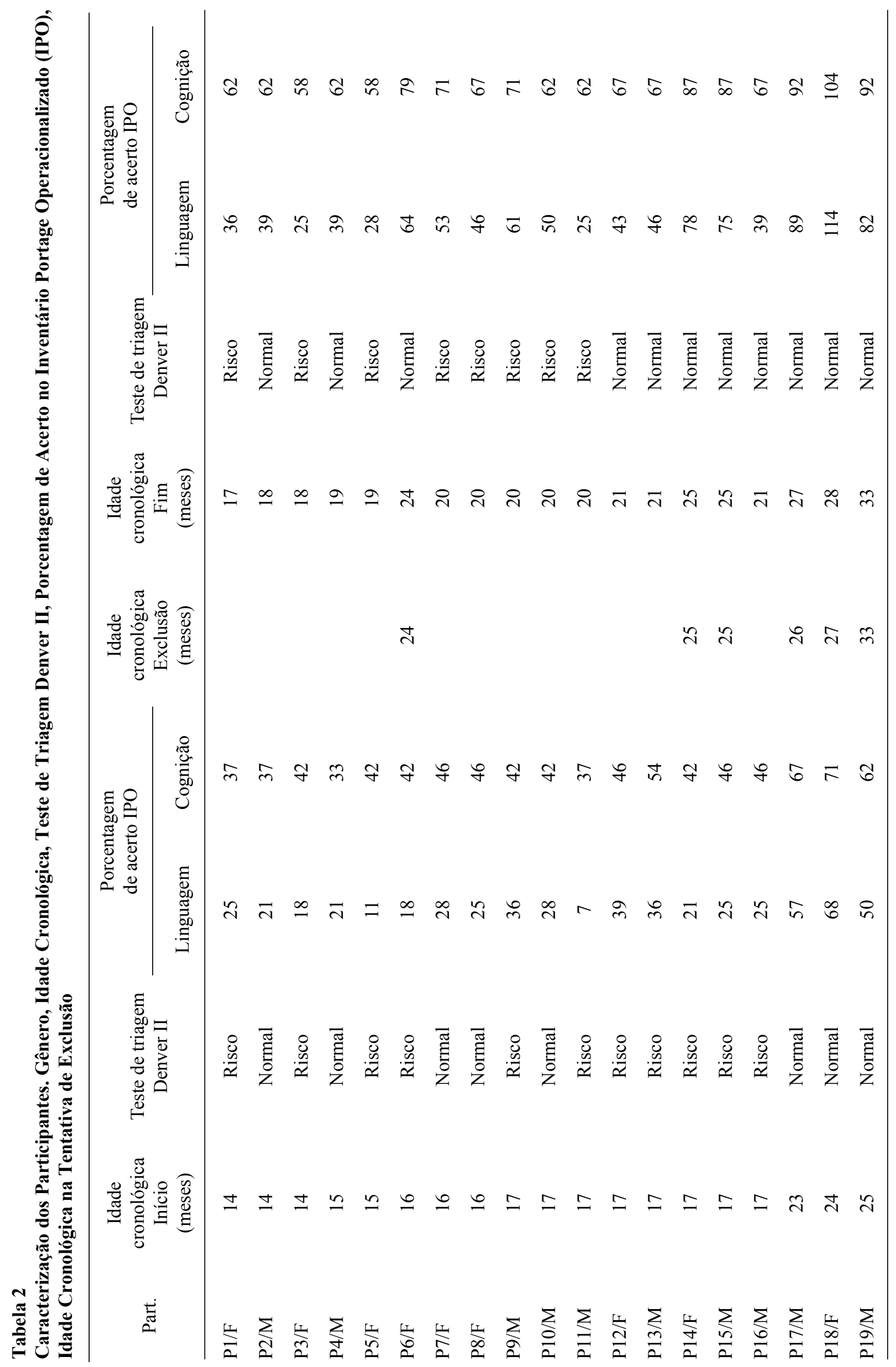


Fase 2 - Ensino de Resposta à Máscara e Ensino das Relações de Linha de Base (LB) com Máscara

Todos os sete participantes (P5, P6, P14, $\mathrm{P} 15, \mathrm{P} 17, \mathrm{P} 18$ e P19) que chegaram a esta fase, aprenderam a escolher a máscara (recipiente opaco), em três sessões (cada estímulo definido foi apresentado seis vezes). Seis participantes (exceto P6) atingiram critério na sessão com dois estímulos de comparação e dois participantes (P14 e P15) não atingiram critério de aprendizagem para as discriminações de linha de base com a máscara nas sessões com três estímulos de comparação, em CRF. Uma participante (P5) não atingiu critério de aprendizagem para a sessão com três estímulos de comparação em VR2 e não foi exposta aos testes por ter saído da creche e encerrado a participação na pesquisa. Os participantes P6, P14, P15 e P19 foram expostos à etapa de sondas mesmo não atingindo o critério, por terem apresentado estabilidade no desempenho e devido à aproximação do final do ano letivo, o que implicaria no encerramento do experimento.

\section{Sondas Controle}

Todos os participantes que chegaram a esta etapa (P6, P14, P15, P17, P18 e P19) atingiram critério nas sondas controle, ou seja, escolheram o objeto definido nas tentativas em que o modelo era uma palavra definida da LB, e os estímulos de comparação eram o objeto definido (CD1 ou $\mathrm{CD} 3$, resposta correta), um objeto indefinido novo (CI7 ou CI8) e a máscara. Nas tentativas em que o modelo era uma palavra ditada indefinida (MI5,/mido/) e os estímulos de comparação eram dois objetos definidos e a máscara, todos os participantes escolheram a máscara.

\section{Fase 3 - Sondas de Exclusão}

As duas tentativas desta sonda verificavam se os participantes selecionariam os objetos indefinidos (CI1 ou CI2) quando as palavras indefinidas (MI1, /fapi/ ou MI2, /beva/) eram apresentadas como modelo. Quatro participantes apresentaram o responder consistente com a exclusão no primeiro bloco para as duas novas relações apresentadas. Dois participantes escolheram por exclusão apenas na segunda sonda. $\mathrm{Na}$ primeira sonda realizada, P6 selecionou o estímulo definido, e P17 selecionou a máscara. Foi realizada uma nova sessão com uma sonda de exclusão para a relação MI1-CI1 (estímulos de comparação: dois objetos definidos e um objeto indefinido), substituindo a máscara por um objeto definido. Nessa ocasião, ambos os participantes atingiram critério para a relação MI1-CI1 (ver Tabela 3).

\section{Sondas de Discriminação}

As duas tentativas desta sonda verificaram se a escolha do participante estaria sob controle da relação que emergiu na sonda de exclusão. Três participantes, P6, P14 e P19, atingiram o critério de aprendizagem das duas relações emergentes.

\section{Sondas de Nomeação}

Nenhum participante apresentou vocalizações correspondentes aos estímulos modelos apresentados nas sondas de exclusão (Tabela 3).

\section{Sondas de Aprendizagem}

A Tabela 3 apresenta as escolhas dos participantes nas três sondas de aprendizagem. $\mathrm{Ne}$ nhum participante demonstrou aprendizagem com base nestas tentativas, ou seja, escolheu o estímulo correto nos três tipos de sondas de aprendizagem. Um participante, P17, escolheu corretamente em dois tipos sondas de aprendizagem para as I1 e I 2 em dois tipos de sondas (aprendizagem 3 e aprendizagem 1). P6 e P19 apresentaram desempenho consistente com aprendizagem da relação M1-I1 em dois tipos de sondas (sondas de aprendizagem 4 e 1 , e 3 e 1 , respectivamente).

\section{Fase 4 - Ensino por Exclusão}

Dos dois participantes que realizaram esta etapa, um participante (P17) atingiu critério nas duas sondas de discriminação após quatro exposições a tentativas de exclusão (uma sonda de exclusão e três tentativas similares à tentativa de exclusão) para cada estímulo indefinido 
Tabela 3

Escolhas dos Participantes nas Sondas de Exclusão, Discriminação, Nomeação e Aprendizagem

\begin{tabular}{|c|c|c|c|c|c|c|c|c|c|}
\hline \multirow[t]{3}{*}{ Part. } & \multicolumn{3}{|c|}{ Exclusão } & \multicolumn{3}{|c|}{ Discriminação } & \multicolumn{3}{|c|}{ Nomeaçáo } \\
\hline & \multicolumn{3}{|c|}{ Indefinido 1} & \multicolumn{3}{|c|}{ Indefinido 1} & \multicolumn{3}{|c|}{ Indefinido 1} \\
\hline & Ind 1 & Def & $\mathrm{M}$ & Ind 1 & Ind 2 & M & \multicolumn{3}{|c|}{ /Fapi/ } \\
\hline P6 & 1 & 1 & 0 & 1 & 0 & 0 & \multicolumn{3}{|c|}{ - } \\
\hline P14 & 1 & 0 & 0 & 1 & 0 & 0 & \multicolumn{3}{|c|}{ - } \\
\hline P15 & 1 & 0 & 0 & 1 & 0 & 0 & \multicolumn{3}{|c|}{ /beu/ } \\
\hline P17 & 1 & 0 & 1 & 0 & 1 & 0 & \multicolumn{3}{|c|}{ /uáu/ } \\
\hline P18 & 1 & 0 & 0 & 1 & 0 & 0 & \multicolumn{3}{|c|}{ /é, eu não sei/ } \\
\hline \multirow[t]{3}{*}{ P19 } & 1 & 0 & 0 & 1 & 0 & 0 & & - & \\
\hline & \multicolumn{3}{|c|}{ Indefinido 2} & \multicolumn{3}{|c|}{ Indefinido 2} & \multicolumn{3}{|c|}{ Indefinido 2} \\
\hline & Ind2 & Def & M & Ind2 & Ind 1 & M & \multicolumn{3}{|c|}{ /Beva/ } \\
\hline P6 & 1 & 0 & 0 & 1 & 0 & 0 & \multicolumn{3}{|c|}{ - } \\
\hline P14 & 1 & 0 & 0 & 1 & 0 & 0 & \multicolumn{3}{|c|}{ /be/ } \\
\hline P15 & 1 & 0 & 0 & 0 & 1 & 0 & \multicolumn{3}{|c|}{ /beu/ } \\
\hline P17 & 1 & 0 & 0 & 0 & 1 & 0 & \multicolumn{3}{|c|}{ /uél } \\
\hline P18 & 1 & 0 & 0 & 0 & 0 & 1 & \multicolumn{3}{|c|}{ /eu não sei, é/ } \\
\hline P19 & 1 & 0 & 0 & 1 & 0 & 0 & \multicolumn{3}{|c|}{-} \\
\hline \multirow[t]{3}{*}{ Part. } & \multicolumn{3}{|c|}{ Aprendizagem 4} & \multicolumn{3}{|c|}{ Aprendizagem 3} & & dizag & \\
\hline & & lefinid & & & lefinid & & & efinid & \\
\hline & Ind 1 & Ind 3 & M & M & Def & Ind7 & M & Def & Ind 1 \\
\hline P6 & 1 & 0 & 0 & 0 & 0 & 1 & 1 & 0 & 0 \\
\hline P14 & 1 & 0 & 0 & 0 & 0 & 1 & 0 & 0 & 1 \\
\hline P15 & 1 & 0 & 0 & 0 & 0 & 1 & 0 & 1 & 0 \\
\hline P17 & 0 & 1 & 0 & 1 & 0 & 0 & 1 & 0 & 0 \\
\hline P18 & 0 & 1 & 0 & 0 & 0 & 1 & 1 & 0 & 0 \\
\hline P19 & 0 & 1 & 0 & 1 & 0 & 0 & 1 & 0 & 0 \\
\hline & & lefinid & & & lefinid & & & efinid & \\
\hline & Ind 2 & Ind 4 & $\mathrm{M}$ & M & Def & Ind8 & $\mathrm{M}$ & Def & Ind 2 \\
\hline P6 & 0 & 1 & 0 & 0 & 1 & 0 & 0 & 1 & 0 \\
\hline P14 & 0 & 1 & 0 & 0 & 0 & 1 & 0 & 0 & 1 \\
\hline P15 & 0 & 1 & 0 & 0 & 0 & 1 & 0 & 1 & 0 \\
\hline P17 & 0 & 0 & 1 & 1 & 0 & 0 & 1 & 0 & 0 \\
\hline P18 & 0 & 1 & 0 & 0 & 0 & 1 & 0 & 0 & 1 \\
\hline P19 & 1 & 0 & 0 & 0 & 1 & 0 & 0 & 1 & 0 \\
\hline
\end{tabular}

Nota. Números em negrito representam a escolha correta para cada sonda. Part.= participantes; Def.= estímulo definido; Ind.= estímulo indefinido; $\mathrm{M}=$ máscara. (-) = ausência de nomeação. 
(I1 e I2). A participante P18 apresentou acerto consistente para a relação 2 nas três sondas de discriminação; porém, não atingiu o critério de aprendizagem do estudo. Com relação às sondas de aprendizagem, nenhum dos dois participantes apresentou acerto nas três sondas em uma mesma sessão.

Nas sondas de nomeação, o participante P17 emitiu a verbalização/beca/ diante dos estímulos I1 e I2 após o primeiro ensino por exclusão, e emitiu as verbalizações /atata/ para I1 e /uataki/ para I 2 após as sondas. A participante P18 emitiu as verbalizações para I1 e I2, respectivamente: /pafi/ e /beco/ após o primeiro ensino; /eu não sei/ e /beva/ após o segundo ensino; e /é beva/ e /é beva/ após o terceiro ensino.

\section{Discussão}

O estudo teve por objetivo avaliar, em crianças com idades entre 14 e 25 meses, a aprendizagem por exclusão de relações entre nomes e objetos empregando sondas de discriminação. Adicionalmente, visou comparar o desempenho dos participantes nas sondas de discriminação com o desempenho nas sondas de aprendizagem e de nomeação. Nesse estudo, dos 19 participantes que iniciaram o experimento, dois atingiram o critério de linha de base e realizaram as etapas de sonda. Quatro participantes (P6, P14, P15 e P19) apresentaram desempenho estável (ainda que não tenham atingido os critérios de aprendizagem de linha de base) e realizaram as etapas de sonda. Dos oito participantes que não atingiram critério da linha de base durante o ensino com dois estímulos de comparação, três apresentaram escolha por preferência de um estímulo específico (P2: D3; P11: D6; e P16: D3) e quatro apresentaram preferência por uma posição específica (P1 e P2: esquerda; P3 e P16: direita). Dos quatro participantes que não atingiram critério no ensino com três estímulos de comparação, três participantes apresentaram preferência por um estímulo (P4: D6; P9: D3 e P13: D3) e um participante respondeu sob controle por posição (P13: centro). O estabelecimento de controle de estímulos por características irrelevantes do arranjo experimental, em detrimento do controle de estímulos programado pelo experimentador, parece ter produzido o desempenho das crianças no ensino das relações de linha de base (Dube \& Mcllvane, 1996).

Os seis participantes que apresentaram estabilidade no desempenho da linha de base obtiveram $100 \%$ de acertos nas sondas controle, atestando a estabilidade das discriminações ensinadas. Este resultado é importante, pois reduz a possibilidade de que as escolhas dos participantes nas sondas subsequentes sejam interpretadas apenas com base no controle pela novidade dos estímulos. Nas sondas de exclusão, observou-se a ocorrência regular do responder por exclusão para os seis participantes, entre 24 e 33 meses, resultados consistentes com os apontados pela literatura (e.g., Antoniazzi et al., 2014; Barbosa et al., 2015; Costa et al., 2013; Costa et al., 2001; Dixon, 1977; Domeniconi et al., 2007; Ribeiro \& Schmidt, 2015; Ribeiro et al., 2016; Schmidt et al., 2016; Wilkinson \& McIlvane, 1997). Além do responder consistente na escolha do objeto indefinido diante do nome indefinido, o responder por exclusão também foi consistentemente observado no outro tipo de sondas controle, nas quais nenhum estímulo indefinido era apresentado e a exclusão dos estímulos definidos resultaria na seleção da máscara (bolso plástico opaco).

Em relação à avaliação de aprendizagem, a hipótese inicial era de que a utilização de sondas de discriminação poderia aumentar a probabilidade de desempenhos consistentes com a aprendizagem depois de apenas uma escolha por exclusão, quando comparadas às outras medidas. A escolha nas sondas de discriminação realizava-se entre as relações indefinidas emergentes por exclusão, com a ausência de estímulos novos ou distratores presentes nas tradicionais sondas de aprendizagem. Como observado, três participantes (P6, P14 e P19) atingiram o critério nas sondas de discriminação, enquanto nenhum deles atingiu o critério nas sondas de aprendizagem tradicionais (acerto em todas as três sondas, nomeadas de aprendizagem 1, 3 e 4). É importante destacar que na exposição à sonda de exclusão e sondas de discriminação, os participantes estavam com 24, 25 e 33 meses, respectivamente, 
com oito meses de diferença do que tinham no início do ensino das relações de linha de base.

Dois participantes (P17 e P18) realizaram a fase de ensino por exclusão e apresentaram acerto para todas as tentativas similares à tentativa de exclusão. Um participante (P17), na ocasião com 27 meses, atingiu critério nas sondas de discriminação após uma tentativa de exclusão e três tentativas similares à tentativa de exclusão. A participante P18, com 28 meses, acertou as três tentativas de sondas de discriminação para a relação I2 /beva/, o que sugere aprendizagem apenas para uma relação (I2).

Os resultados indicam que as sondas de discriminação, realizadas após as tentativas de responder por exclusão (de Rose et al., 1996; de Rose et al., 1989; Dixon, 1977; Mcllvane \& Stoddard, 1981), apresentaram-se como alternativa viável para medir a aprendizagem das relações de discriminações condicionais emergentes. É importante ressaltar que os parâmetros que permitem a escolha por exclusão não são apresentados nessas tentativas (comparações definidos da linha de base). Ainda assim, os quatro participantes (P6, P14, P17 e P19) responderam corretamente às sondas de discriminação indicando a aquisição de uma nova relação nome-objeto sem ensino prévio.

Por outro lado, de acordo com a análise realizada por Costa et al. (2014), no cômputo do desempenho de todos os participantes abaixo dos 36 meses, 10 das 103 crianças $(9,7 \%)$ apresentaram resultados indicativos de aprendizagem (acerto nas três sondas 1, 2 e 3, ou sondas 4, 3 e 1 de aprendizagem): um em seis participantes no estudo de Domeniconi et al. (2007), uma em 10 crianças no estudo de Antoniazzi et al. (2014), um em 10 participantes no estudo de Barbosa et al. (2015), uma em 21 crianças no estudo de Ribeiro et al. (2016), e seis em 56 participantes no estudo de Schmidt et al. (2016), até a faixa etária de 36 meses. Geralmente, os participantes apresentam acertos em uma ou duas sondas (e.g., Ribeiro et al., 2016; Schmidt et al., 2016). Este padrão também foi observado neste estudo, ao replicar os resultados encontrados na literatura e ampliar a amostra de participantes. A taxa de acertos em cada sonda, na Fase 3, foi de $41 \%$ para a aprendizagem $1,25 \%$ para a aprendizagem 3 e 33\% para a aprendizagem 4, indicando melhor desempenho no controle por rejeição. A análise dos padrões de erro nestas sondas indicou que sete dos nove erros apresentados na sonda de aprendizagem 3 se referiram à escolha do objeto indefinido mais novo (Indefinido 7 ou Indefinido 8). Da mesma forma, sete dos oito erros nas sondas de aprendizagem 4 foram escolhas do Indefinido 3 ou Indefinido 4. Resultados semelhantes foram observados em outros estudos com crianças de mesma idade (e.g., Ribeiro de Souza et al., 2018; Schmidt et al., 2016) e corroboram as evidências de que o controle pela novidade exerce influência sobre os padrões de escolha de crianças em tarefas análogas (Horst et al., 2011).

Ainda que quatro participantes (P6, P14, P17 e P19) apresentassem aprendizagem das relações emergentes por exclusão, a aprendizagem das relações condicionais auditivo-visuais não foi condição suficiente para que os participantes nomeassem os objetos. Nas sondas de nomeação (respostas de tato, Skinner, 1957), nenhum participante nomeou os estímulos. E para os que os nomearam, não foi condição suficiente para a precisão de topografia na nomeação. Na nomeação o controle exigido é pela relação entre a palavra ditada e o objeto, sendo que o objeto está presente. As duas palavras referentes às relações emergentes compartilhavam diferenças múltiplas (diferenças entre mais da metade dos fonemas), e os objetos compartilhavam diferenças múltiplas (cores e formato), ao invés de palavras e objetos com diferenças críticas, que poderiam aumentar o grau de complexidade da tarefa (Souza, Almeida-Verdu, \& Belivacqua, 2013). Os participantes P17 e P18 apresentaram verbalizações parcialmente correspondentes nas sessões de ensino da relação nome-objeto emergente (P17 diz: /beca/ para I2; /pafi/ para I1 e P18 diz:/beco/ para I2). Esses resultados são confirmados pela literatura: a fala, principalmente de crianças pequenas, não corresponde ou corresponde apenas parcialmente à palavra-modelo (Costa et al., 2014). Pesquisas futuras poderiam investigar se, e sob quais condições, as sondas de nomeação podem auxiliar a monitorar as re- 
lações de controle presentes na aprendizagem de relações emergentes por exclusão.

Os resultados sugerem que as sondas de discriminação podem servir para compor uma proposta de avaliação da aprendizagem por exclusão menos sujeita a interferências de outras fontes de controle (estímulos de comparação das relações que emergiram na exclusão, sem a presença de estímulos novos ou distratores, como nas tradicionais sondas de aprendizagem). Entretanto, novos estudos precisam ser realizados para assegurar a confiabilidade do procedimento. É importante salientar que o número de sondas usadas neste estudo foi comparativamente reduzido em relação ao de estudos anteriores (de Rose et al., 1996; de Rose et al., 1989; Dixon, 1977; McIlvane \& Stoddard, 1981). Consequentemente, estudos futuros devem empregar um número maior de tentativas de avaliação de aprendizagem para reduzir a possibilidade de que os desempenhos observados ocorram ao acaso. Adicionalmente, considerando as rápidas mudanças no repertório dos participantes nesta faixa etária, verificou-se o aumento na pontuação de cada um dos participantes da primeira para a última avaliação do IPO, e a redução de participantes com indicativo de risco para o desenvolvimento no Denver II, e futuras investigações devem avaliar se os instrumentos utilizados se mostraram preditores dos resultados produzidos pelos participantes.

\section{Referências}

Almeida, C. G. M. (2014). Novos repertórios verbais em crianças pequenas: ouvinte, falante e classes de estímulos equivalentes. (Tese de doutorado). Recuperado em https://repositorio.ufscar.br/ handle/ufscar/5995

Antoniazzi, M., Domeniconi, C., \& Schmidt, A. (2014). Efeito da pré-exposição ao objeto no desempenho por exclusão e na aprendizagem da relação nome-objeto. Acta Comportamentalia, 22(1), 23-36. Recuperado em http://pepsic. bvsalud.org/scielo.php?script=sci_arttext\&pid $=\mathrm{S} 0188-81452014000100003 \& \operatorname{lng}=\mathrm{pt} \& \mathrm{t} \operatorname{lng}=\mathrm{pt}$

Barbosa, B. F., Gomes, G. F., Costa, A. R. A., \& Schmidt, A. (2015). Aprendizagem de discriminação auditivo-visual a partir de uma única tentativa de exclusão com objetos tridimensionais.
Revista Brasileira de Terapia Comportamental e Cognitiva, 17(3), 22-37. Recuperado em http:// www.usp.br/rbtcc/index.php/RBTCC/article/ view/813

Bion, R. A. H., Borovsky, A., \& Fernald, A. (2013). Fast mapping, slow learning: Disambiguation of novel word-object mappings in relation to vocabulary learning at 18,24 , and 30 months. Cognition, 126, 39-53. doi: https://doi.org/10.1016/j. cognition.2012.08.008

Bluma, S., Shearer, M., Frohman, A., \& Hilliard, J. (1976). Portage guide to early education. Portage, WI: Cooperative Educational Service Agency.

Carr, D. (2003). Effects of exemplar training in exclusion responding on auditory-visual discrimination tasks with children with autism. Journal of Applied Behavior Analysis, 36, 507-524. doi: 10.1901/jaba.2003.36-507

Costa, A. R. A., Domeniconi, C., \& de Souza, D. G. (2014). Controle de estímulos, mapeamento simbólico emergente e aquisição de vocabulário. In J. C. de Rose, M. S. C. A. Gil, \& D. G. de Souza (Eds.), Comportamento simbólico: Bases conceituais e empíricas (pp. 269-308). Marília, SP: Oficina Universitária.

Costa, A. R. A., Grisante, P. C., Domeniconi, C., de Rose, J. C., \& de Souza, D. G. (2013). Naming new stimuli after selection by exclusion. Paidéia (Ribeirão Preto), 23(55), 217-224. doi: 10.1590/1982-43272355201309

Costa, A. R. A., Wilkinson, K. M., McIlvane, J. W., $\&$ de Souza, D. G. (2001). Emergent word object mapping by children: Further studies using the blank comparison technique. The Psychological Record, 51(3), 343-355. Retrieved from http://opensiuc.lib.siu.edu/cgi/viewcontent. cgi?article $=1321 \&$ context $=$ tpr

De Rose, J. C., de Souza, D. G., \& Hanna, E. S. (1996). Teaching reading and spelling: Exclusion and stimulus equivalence. Journal of Applied Behavior Analysis, 29, 451-469. doi: 10.1901/jaba.1996.29-451

De Rose, J. C., de Souza, D. G., Rossito, A. L., \& de Rose, T. M. S. (1989). Aquisição de leitura após história de fracasso escolar: Equivalência de estímulos e generalização. Psicologia: Teoria e Pesquisa, 5(3), 325-346. Recuperado em https://revistaptp.unb.br/index.php/ptp/article/ view/1384/376 
Dixon, L. S. (1977). The nature of control by spoken words over visual stimulus selection. Journal of the Experimental Analysis of Behavior, 27(3), 433-442. doi: 10.1901/jeab.1977.27-433

Domeniconi, C., Costa, A. R. A., de Souza, D. G., $\&$ de Rose, J. C. (2007). Responder por exclusão em crianças de 2 a 3 anos em uma situação de brincadeira. Psicologia: Reflexão $e$ Crítica, 20(2), 342-350. doi: 10.1590/S010279722007000200021

Dube, W. V., \& Mcllvane, W. J. (1996). Some implications of a stimulus control topography analysis for emergent stimulus classes. In T. R. Zentall \& P. M. Smeets (Eds.). Stimulus class formation in humans and animals (pp. 197-218). North-Holland, Netherlands: Elsevier.

Frankenburg, K.W., Dodds, J., Archer, P., \& Bresnick, B. (1990). Denver II: Technical manual. Denver, CO: Denver Developmental Materials.

Gil, M. S. C. A., Oliveira, T. P., de Sousa, N. M., \& Faleiros, D. A. M. (2006). Variáveis no ensino de discriminação para bebês. Psicologia: Teoria e Pesquisa, 22(2), 143-152. doi: 10.1590/ S0102-37722006000200003

Greer, R. D., \& Du, L. (2015). Experience and the onset of the capability to learn names incidentally by exclusion. The Psychological Record, 65(2), 355-373. doi: 10.1007/ s40732-014-0111-2

Horst, J. S., \& Samuelson, L. K. (2008). Fast mapping but poor retention by 24-monthold infants. Infancy, 13(2), 128-157. doi: $10.1080 / 15250000701795598$

Horst, J. S., Samuelson, L. K., Kucker, S. C., \& McMurray, B. (2011). What's new? Children prefer novelty in referent selection. Cognition, 118(2), 234-244. doi: 10.1016/j.cognition.2010.10.015

Kazdin, A. E. (1982). Single-case research designs: Methods for clinical and applied settings. New York: Oxford University Press.

Lamprecht, R. R., Bonilha, G. F. G., Freitas, G. C. M., Matzenauer, C. L. B. Mezzomo, C. L., Oliveira, C. C., \& Ribas, L. P. (2004). Aquisição Fonológica do Português: Perfil de desenvolvimento e subsidios para terapia. São Paulo, SP: Artmed.

Langsdorff, L. C., Schmidt, A., \& Domeniconi, C. (2015). Aprendizagem de relações auditivo-visuais por meio de tentativas de exclusão. Interação em Psicologia, 19, 25-35. doi: 10.5380/psi. v19i1.33978
McIlvane, W. J., \& Stoddard, T. (1981). Acquisition of matching to sample performances in severe retardation: Learning by exclusion. Journal of Mental Deficiency Research, 25, 33-48.

Pedromônico, M. R. N., Bragatto, E. L., \& Strobilus, R. (1999). Teste de Triagem Denver II. São Paulo, SP: Universidade Federal de São Paulo.

Ribeiro, J., \& Schmidt, A. (2015). Aprendizagem de relações palavra-objeto por bebês em um procedimento de introdução sucessiva de estímulos. Revista Brasileira de Análise do Comportamento, 11(1), 70-79. doi: 10.18542/rebac.v11i1.3777

Ribeiro, T. A., Gallano, T. P., Souza, D. H., \& de Souza, D. G. (2016). Responding and Learning by Exclusion in 2-Year-Olds: The Case of Adjectives. The Psychological Record, 1, 1-22. doi: 10.1007/s40732-016-0213-0

Ribeiro de Souza, L. M., Gil, M. S. C. A., \& Garcia, L. T. (2018). Aprendizagem por exclusão em crianças pequenas. Paidéia (Ribeirão Preto), 28, e2810. doi: http://dx.doi.org/10.1590/1982$-4327 \mathrm{e} 2810$

Ribeiro de Souza, L. M., Minto de Sousa, N., \& Gil, M. S. C. A. (2016). Aprendizagem de relações emergentes por exclusão em crianças de 2 a 3 anos. Revista Brasileira de Análise do Comportamento, 12, 44-53. doi: http://dx.doi. org/10.18542/rebac.v12i1.3788

Schmidt, A., Franco, M. G. O., Lotério, L. S., \& Gomes, G. F. (2016). Learning name-object relations after a single exclusion trial in 18- to 48-month-old children. The Psychological Record, 66, 53-63. doi: 10.1007/s40732-015-01512

Sertori, N. M. (2013). Discriminações condicionais em bebês de risco: O responder por exclusão. (Dissertação de mestrado). Recuperado em https://repositorio.ufscar.br/handle/ufscar/3143

Skinner, B. F. (1957). Verbal Behavior. New York: Appleton-Century-Crofts.

Souza, F. C., Almeida-Verdu, A. C. M., \& Bevilacqua, M. C. (2013). Ecoico e nomeação de figuras em crianças com deficiência auditiva pré-lingual com implante coclear. Acta Comportamentalia, 21(3), 325-339. Recuperado em http:// www.revistas.unam.mx/index.php/acom/article/ view/41749

Wilkinson, K. M., Dube, W. V., \& McIlvane, W. J. (1998). Fast mapping and exclusion (emergent matching) in developmental language, behav- 
ior analysis, and animal cognition research. The Psychological Record, 48, 407-422. doi: 10.1007/BF03395281

Wilkinson, K. M., \& McIlvane, W. J. (1997). Blank comparison analysis of emergent symbolic mapping by young children. Journal of Experimental Child Psychology, 67(2), 115-130. doi: 10.1006/ jecp.1997.2402
Williams, L. C. A., \& Aiello, A. L. R. (2001). O Inventário Portage Operacionalizado: Intervenção com famílias. São Paulo, SP: Memnon.

Recebido: $17 / 11 / 2017$

$1^{a}$ revisão: $11 / 02 / 2018$

$2^{a}$ revisão: $14 / 02 / 2018$

Aceite final: $14 / 02 / 2018$ 